\title{
The contribution of secondary eclipses as astrophysical false positives to exoplanet transit surveys
}

\author{
A. Santerne ${ }^{1}$, F. Fressin ${ }^{2}$, R. F. Díaz ${ }^{3}$, P. Figueira ${ }^{1}$, J.-M. Almenara ${ }^{3}$, and N. C. Santos ${ }^{1,4}$ \\ ${ }^{1}$ Centro de Astrofísica, Universidade do Porto, Rua das Estrelas, 4150-762 Porto, Portugal \\ e-mail: alexandre.santerne@astro.up.pt \\ 2 Harvard-Smithsonian Center for Astrophysics, Cambridge, MA 02138, USA \\ 3 Aix Marseille Université, CNRS, LAM (Laboratoire d'Astrophysique de Marseille) UMR 7326, 13388 Marseille, France \\ ${ }^{4}$ Departamento de Física e Astronomia, Faculdade de Ciências, Universidade do Porto, Portugal
}

Received 14 March 2013 / Accepted 13 July 2013

\section{ABSTRACT}

\begin{abstract}
We investigate the astrophysical false-positive configuration in exoplanet-transit surveys. It involves eclipsing binaries and giant planets that present only a secondary eclipse, as seen from the Earth. To test how an eclipsing binary configuration can mimic a planetary transit, we generated synthetic light curves of three examples of secondary-only eclipsing binary systems that we fit with a circular planetary model. Then, to evaluate its occurrence we modeled a population of binaries in double and triple systems based on binary statistics and occurrence. We find that $0.061 \% \pm 0.017 \%$ of main-sequence binary stars are secondary-only eclipsing binaries that mimics a planetary transit candidate with a size down to the size of the Earth. We then evaluate the occurrence that an occultingonly giant planet can mimic an Earth-like planet or even a smaller one. We find that $0.009 \% \pm 0.002 \%$ of stars harbor a giant planet that only presents the secondary transit. Occulting-only giant planets mimic planets that are smaller than the Earth, and they are in the scope of space missions like Kepler and PLATO. We estimate that up to $43.1 \pm 5.6$ Kepler objects of interest can be mimicked by this configuration of false positives, thereby re-evaluating the global false-positive rate of the Kepler mission from $9.4 \pm 0.9 \%$ to $11.3 \pm 1.1 \%$. We note, however, that this new false-positive scenario occurs at relatively long orbital periods compared with the median period of Kepler candidates.
\end{abstract}

Key words. planetary systems - techniques: photometric - binaries: eclipsing

\section{Introduction}

Many astrophysical false positives can mimic an exoplanetary transit. These astrophysical false positives are composed of various configurations of eclipsing binaries (EB) in which the companion star is gravitationally bound to the target star (EB in double or triple systems) or in the background or foreground within the photometric aperture of the instrument (Almenara et al. 2009). A transit of a small planet may also be explained by the transit of a larger planet orbiting a background star or a stellar companion of the target star. Recently, Fressin et al. (2013) have investigated the rate of each false positive scenario in the stellar population of the Kepler field. By simulating the Kepler transit survey using assumptions that are as realistic as possible, they find that the global false-positive rate of Kepler is $9.4 \% \pm 0.9 \%$. The authors find that most of false positives involve Neptune-size planets transiting companion stars of the target and mimicking earth-size ones. Their analysis re-evaluates the overall false-positive probability of Kepler previously estimated as $\sim 5 \%$ by Morton \& Johnson (2011), which was later found to fall short of the direct estimates by spectroscopic observations (Santerne et al. 2012). An accurate estimation of the false positive rate is crucial to determining the occurrence and properties of extrasolar planets from the list of potential transiting planets (Batalha et al. 2012). Such studies of planet occurrence and properties in the Kepler field have been performed by Howard et al. (2012), but they assume that the rate of false positives could be neglected. These results could be affected if this is not the case, as in fact observed for giant planets, for which both a direct spectroscopic survey (Santerne et al. 2012) and a dedicated statistical analysis of the detections (Fressin et al. 2013), converge towards a false positive rate between $17.7 \%$ and $35 \%$.

The limitations of current spectrographs do not allow the establishment of the smallest CoRoT and Kepler planets using the usual radial velocity technique. The planet-validation technique is a new method of establishing the planetary nature of a transiting planet candidate (e.g. Torres et al. 2011). It consists in computing the probability that each false positive scenario has to reproduce the observed data sets. Then, these probabilities are compared with the probability that the transit signal is produced by a bona-fide and undiluted planet. A planet is thus considered as "validated" if the planet scenario is significantly the most likely one. An exhaustive set of astrophysical false positive scenarios must be considered in this process to avoid underestimating the false-positive probability of the candidate.

The list of currently considered astrophysical false-positive scenarios is explained well in Cameron (2012) and Fressin et al. (2013). Briefly, a planetary transit might be mimicked by

1. an eclipse of an FGK-type main-sequence star by a low-mass star or a brown dwarf with a radius similar to that of Jupiter;

2. an eclipse of a giant star by a main-sequence star;

3. a grazing EB;

4. an eclipse of a binary in the foreground or background, aligned with the target star, as seen from the Earth;

5. an eclipse of a binary bound with the target star; 


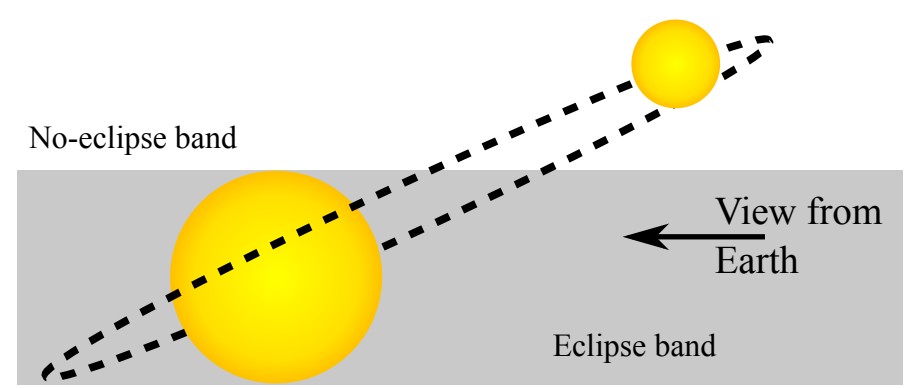

Fig. 1. Sketch of the configuration where a binary only shows a secondary eclipse.

6. a transit of a planet on a star aligned with the target star, as seen from the Earth, in the foreground or background;

7. a transit of a planet on a star physically bound with the target star;

8. a transiting or occulting white dwarf.

While the first three scenarios can be easily distinguished by a radial velocity follow-up (Santerne et al. 2012), the last ones require rigorous investigations to be statistically rejected. We note that these scenarios may also be constrained thanks to radial velocity diagnosis (Santerne et al., in prep.).

We present the false-positive scenario configuration involving secondary-only eclipsing binaries or giant planets. This false-positive scenario is only considered in the false-positive probability estimation of Morton \& Johnson (2011) and planetvalidation process described in Morton (2012) and Dawson et al. (2012) and is not considered in Kepler planet validation and in the false-positive probability estimation of Fressin et al. (2013). We first present the configuration secondary-only eclipsing binary in Sect. 2 and evaluate its occurrence by simulating a population of eclipsing binaries in Sect. 3. We then consider the same configuration in the case of a giant planet and evaluate its occurrence in Sect. 4. Finally, we discuss its impact on exoplanet-transit surveys like CoRoT and Kepler in Sect. 5.

\section{Secondary-only eclipsing binaries as false positive scenario}

\subsection{Conditions for secondary-only eclipsing binaries}

In all cases where the planet-validation technique was used to establish the planetary nature (e.g., Fressin et al. 2012a,b), only the primary eclipse - i.e., when the smaller object passes in front of the larger object - of EB were considered as the cause of the observed transit. In some geometrical configurations, an EB might not present a primary eclipse, but only a secondary eclipse - i.e. when the smaller object is eclipsed by the larger one (see Fig. 1). This configuration was accounted for only in the false-positive studies of Morton \& Johnson (2011), Morton (2012), and Dawson et al. (2012), but they do not provide any detailed statistics or occurrence rate. For the first time, Santerne et al. (2012) characterized two eclipsing binaries among the Kepler transiting exoplanets candidates (namely KOI-419 and KOI-698) that only present their secondary eclipse, as seen from the Earth. A secondary eclipse of EBs can be as shallow as to mimic a transit event of a planet (Santerne et al. 2012, see also
Fig. 4). This configuration is only possible for orbits for which the impact parameters $\left(b_{\text {prim }}\right.$ and $\left.b_{\text {sec }}\right)$ satisfy (Winn 2010)

$$
\begin{gathered}
b_{\text {prim }}=\frac{a}{R_{1}} \cos (i)\left(\frac{1-e^{2}}{1+e \sin \omega}\right)>1+\frac{R_{2}}{R_{1}}, \\
b_{\text {sec }}=\frac{a}{R_{1}} \cos (i)\left(\frac{1-e^{2}}{1-e \sin \omega}\right)<1+\frac{R_{2}}{R_{1}},
\end{gathered}
$$

where $a$ is the semi-major axis, $R_{1}$ and $R_{2}$ the radii of the primary and secondary stars (respectively), $i$ the orbital inclination, $e$ the orbital eccentricity, and $\omega$ the argument of periastron. To present only a secondary eclipse, the orbit should have nonzero eccentricity, with an argument of periastron $\omega \in\left[180^{\circ} ; 360^{\circ}\right]$ and an orbital inclination different from $90^{\circ}$. The eccentricity of the system is the main parameter driving this false-positive configuration.

\subsection{Eccentricity of short-period binaries}

It has been reported by Duquennoy \& Mayor (1991), Halbwachs et al. (2003), and Raghavan et al. (2010) that main-sequence eccentric binaries do not have orbital periods of less than about ten days. This circularization period is compatible with the theory of the tidal evolution of close-in binaries (Zahn 1977, 1989). On the other hand, numerous detached-EB from the Kepler catalog (Slawson et al. 2011) are eccentric with orbital periods down to about four days (see for example KIC4947726 and KIC4753561 in the Kepler EB catalog). A thorough analysis of EB in this catalog can reveal their distribution of eccentricities, which is beyond the scope of this paper. This has been performed based on the Trans-Atlantic Exoplanet Surveys (Devor et al. 2008) that confirm the nonzero eccentricity of some eclipsing binaries with orbital period shorter than ten days (as displayed in Fig. 2). However, significant eccentricity values in such short-period binary system have been reported for only a very few cases. Indeed, among the 827 EBs (respectively 725) reported by Devor et al. (2008) with orbital period of less than ten days (respectively, five days), only five systems (respectively three systems) present a significant eccentricity $(>3-\sigma)$. This corresponds to $0.6 \pm 0.3 \%$ of their total sample of EBs (respectively, $0.4 \pm 0.2 \%$ ). Moreover, we cannot exclude that these systems are actually orbiting in a wider stellar system in which the Kozai mechanism (Kozai 1962) can counterbalance the tidal circularization.

On the other hand, Bulut \& Demircan (2007) compiled a catalog of 124 eccentric eclipsing binaries and 150 candidates reported in the HiPPARcos catalog (Perryman et al. 1997), the atlas of (O-C) diagram of Kreiner et al. (2001) and the ninth cata$\log$ of spectroscopic binary orbits $\left(S_{B^{9}}\right.$, Pourbaix et al. 2004). The majority of these binaries have an orbital period of less than ten days (see Fig. 2). From the $S_{B^{9}}$ catalog, $16 \%$ of spectroscopic binaries present an orbital period of less than ten days and a significant eccentricity (assumed $>0.1$ ) among 1515 systems of various spectral types and luminosity classes. We supposed that these eccentric short-period binaries might be dominated by systems with a massive primary with no convective zone to dissipate tidal forces. Those systems might also be younger than the circularization timescale. Abt (2005) collected nearly 400 systems from $S_{B^{9}}$ having a B0-F0 V-IV primary. Sixty percent (respectively, 23\%) of such systems have an eccentricity greater than zero (respectively, greater than 0.1). Binaries with massive primary are thus less circularized than the population of binaries reported in $S_{B^{9}}$. Since transit surveys focus mainly on $\operatorname{FGK}(\mathrm{M})$ dwarfs, we therefore assume that this configuration 


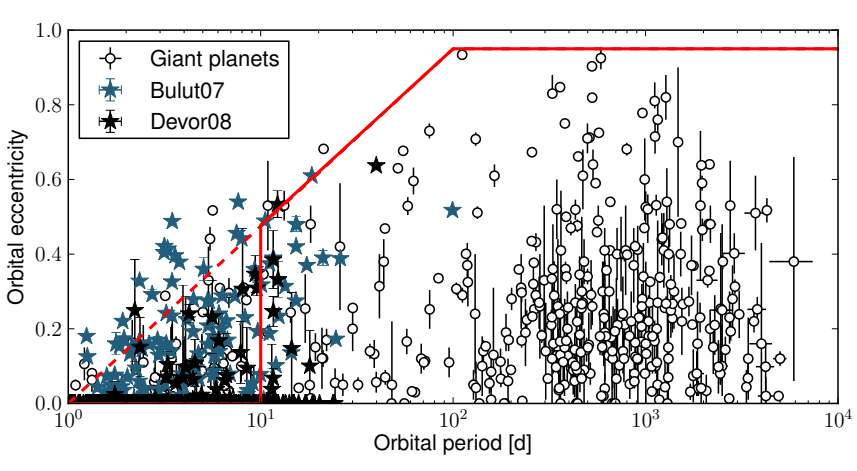

Fig. 2. Period-Eccentricity diagram of giant planets discovered by radial velocity (open circles) and eclipsing binaries from TrES (black stars, Devor et al. 2008) from the catalog of eccentric eclipsing binaires (blue stars, Bulut \& Demircan 2007). The solid red line displays the assumed upper-envelope of the eccentricity of binaries, and the dashed red line, the upper-envelope of eccentricity of giant planets.

of EB can occur at any orbital period, but is relatively rare for orbital periods less than ten days.

\subsection{Shape of secondary-only eclipse}

Secondary-only eclipses can easily mimic the depth of a planetary transit as shown with KOI-419 and KOI-698 (Santerne et al. 2012). Their transit shapes are either "V-shaped" (grazing eclipses, see Fig. 3) or do not have limb-darkening effects (total eclipses). In all cases, the transit-like event has a relatively short duration (see Fig. 4). However, several planets have already been reported with short and grazing transits, e.g. CoRoT-10 b (Bonomo et al. 2010), showing that V-shape transit can be compatible with planets.

To further test how a secondary-only EB can mimic a planetary transit, we generated synthetic light curves of three binary systems with the PASTIS code (Díaz et al. 2013) for the Kepler bandpass, using the Ebop code (Southworth 2008, and references therein) and stellar atmosphere models from the PHOENIX/BT-Settl library (Allard et al. 2012). For the three systems, we assumed an orbital period of $\sim 63.1$ days, inclination of $89^{\circ}$, eccentricity of 0.3 , and argument of periastron of $270^{\circ}$. These values are intended to represent the median values of the distribution of secondary-only eclipsing binaries (see Fig. 4 and Sect. 3.3). For the first system, we assumed two stars with masses of $1 M_{\odot}$ and $0.5 M_{\odot}$ that produce a secondaryonly eclipse at the level of $\sim 1 \%$. For the second system, we assumed two stars with masses of $1 M_{\odot}$ and $0.2 M_{\odot}$ that produce a secondary-only eclipse at the level of $\sim 0.1 \%$. Finally, the third system is composed of two stars of $1 M_{\odot}$ for which one host a secondary-only eclipsing companion of $0.5 M_{\odot}$. This system produces a diluted secondary-only eclipse at the level of $~ 500 \mathrm{ppm}$. We assumed only white noise with an amplitude of $250 \mathrm{ppm}$, which is the typical precision of the Kepler machine for a magnitude $K_{\mathrm{p}}=15$ target. Synthetic light curves have an integrated sampling of $30 \mathrm{~min}$ to reproduce the Kepler long-cadence data and a timescale of 3.5 years. Synthetic light curves are displayed in Fig. 3.

We fit the three generated light curves with a planetary scenario using the Markov chain Monte Carlo (MCMC) algorithm of the PASTIS code (Díaz et al. 2013) that includes a principal component analysis decomposition to better explore the correlated parameter space. We fixed the eccentricity to zero and
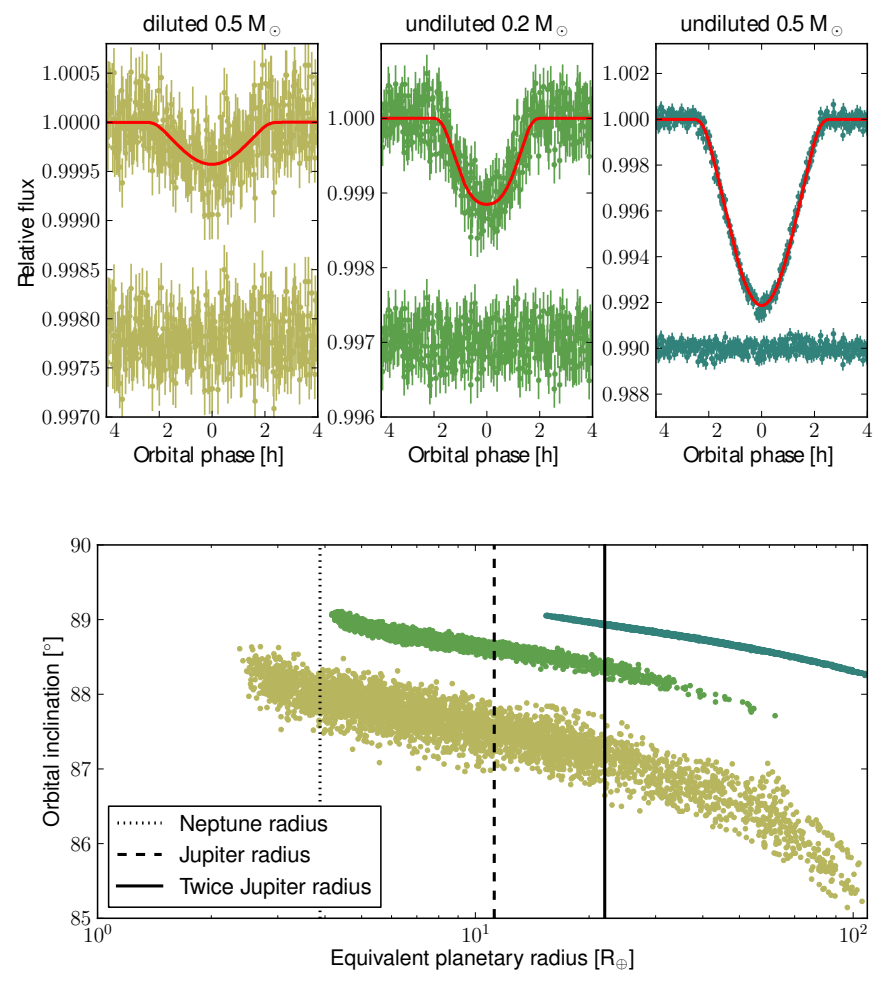

Fig. 3. Top: synthetic Kepler light curve of secondary-only EB (as described in the text) with their best planetary model and residuals. From left to right, light curve are generated assuming the $1-0.5 M_{\odot}$ secondary-only EB diluted by a gravitationally bound $1 M_{\odot}$ star, the undiluted 1-0.2 $M_{\odot}$ secondary-only EB and the undiluted $1 M_{\odot}-$ $0.5 M_{\odot}$ secondary-only EB. Bottom: posterior distribution from the MCMC analysis of the aforementioned secondary-only EBs, displaying the correlation between the orbital inclination and the planetary radius (assuming a 1- $R_{\odot}$ host). Colors and ranking from left to right are the same as for the upper plot. Vertical lines indicate the radius of Neptune (dotted line), the radius of Jupiter (dashed line), and twice the radius of Jupiter (solid line).

the limb darkening values to the solar ones from the table of Claret et al. (2012). Only the orbital period, transit epoch, system scale $\left(a / R_{\star}\right)$, radius ratio, orbital inclination, and out-of-transit flux were left as free parameters in the MCMC analysis. In Fig. 3 (bottom) we represent the correlation between the orbital inclination and the measured planetary radius from the posterior distribution of the MCMC analysis, assuming a $1-R_{\odot}$ stellar host. The MCMC fit converged toward a stellar density lower than the Sun, which is still compatible with the values observed for transiting planets (Tingley et al. 2011). This lower stellar density can also be explained by an eccentric planet (Dawson \& Johnson 2012; Dawson et al. 2012). The three synthetic light curves of secondary-only EB are thus compatible with a planet with a radius below twice the one of Jupiter. We note that above the commonly-used $2-R_{\text {Jup }}$ limit for giant planets, objects are most likely of stellar origin and cannot be fit assuming a nonself-emitting object as in case of a planet. The MCMC analysis thus explored unphysical models above the $2 R_{\text {jup }}$-limit. The goal here was to convince skeptical readers that the degeneracy between radius ratio and orbital inclination in case of a $\mathrm{V}$-shape transit allow grazing eclipsing binary to mimic planets. The best planetary models that satisfy $r_{\mathrm{p}}<2 R_{\text {Jup }}$ are displayed in Fig. 3 (top), with their residuals. 

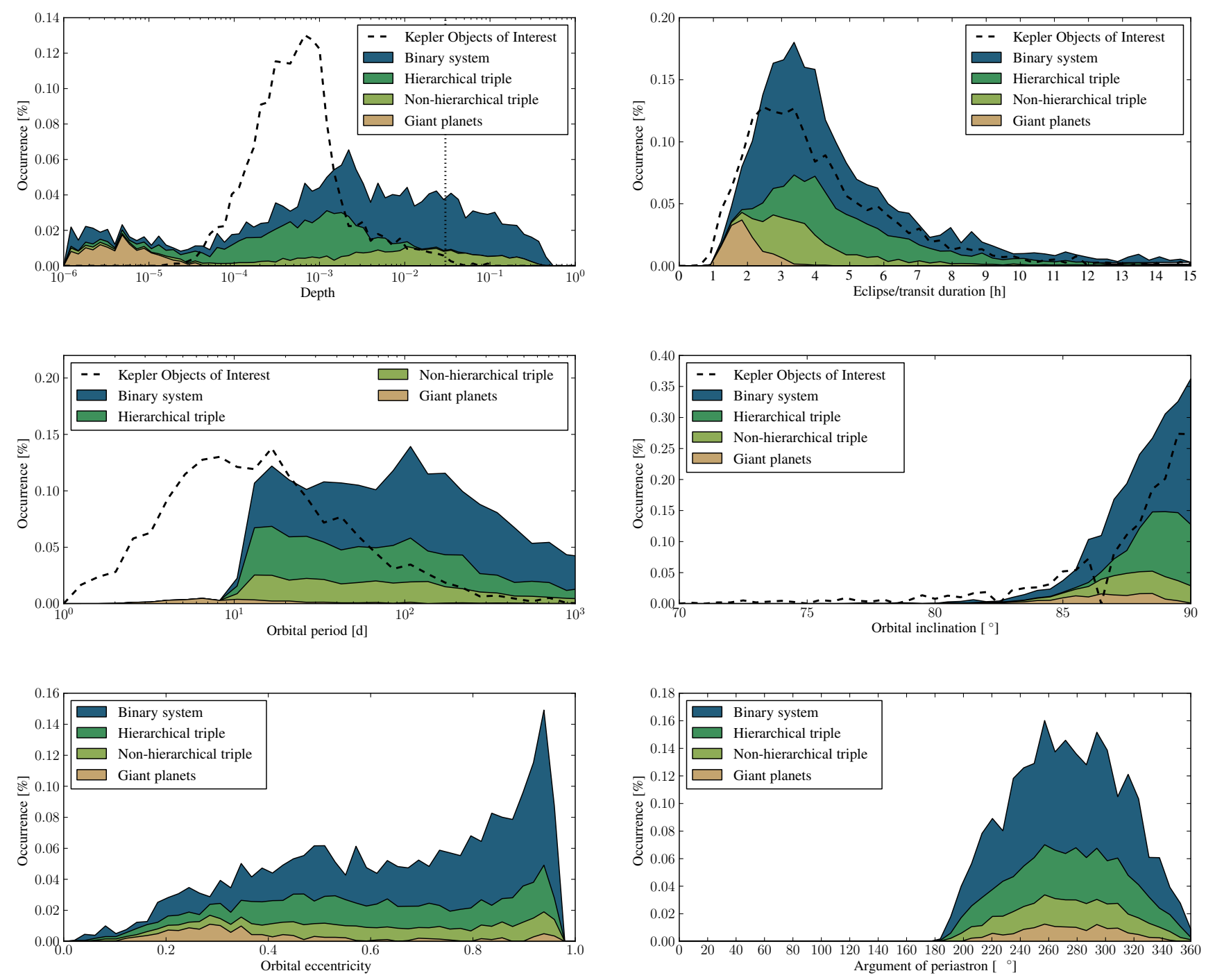

Fig. 4. Stacked distributions (magnified by 30) of the secondary-only EB and occulting-only giant planets for their eclipse/transit depth (upperleft plot), eclipse/transit duration (upper-right plot), orbital period (middle-left plot), inclination (middle-right plot), eccentricity (lower-left plot), and argument of periastron (lower-right plot). The distribution have been normalized to represent the relative occurrence as computed in Table 1. Corresponding distributions of Kepler objects of interest (from Batalha et al. 2012, dashed black line) are also displayed, when known. The vertical dotted line in the upper-left plot represent the commonly-used 3\% upper limit in depth of planetary transit candidates.

From the transit parameters of Batalha et al. (2012), we find that about $60 \%$ (respectively, $92 \%$ ) of the Kepler objects of interest (KOIs) have an impact parameter greater than one within 1- $\sigma$ (respectively, 3- $\sigma$ ). Since the majority of the KOIs are compatible with a grazing transit or V-shape transit within 3- $\sigma$, we therefore do not consider the shape of a transit event as a systematic indication of false positive. We stress that Kepler has a photometric precision high enough to detect the transit of very small candidates. Unfortunately, the signal-to-noise reached by Kepler is too low for the majority of the KOIs to determine the shape of the transit, especially if the candidate is small, the orbital period is long, the host star is active and/or the host star is faint. This does not mean that the majority of the KOIs are actually V-shaped transit.

Beaming, ellipsoidal, and reflexion effects (Mazeh \& Faigler 2010) might be used to identifying eclipsing binary that would produce out-of-transit variations. However, as shown by Shporer et al. (2011), the amplitude of these effects drastically decrease with the orbital period: the beaming effect decreases as $a^{-1 / 2}$, the ellipsoidal effect decreases as $a^{-3}$, and the reflexion effect as $a^{-2}$ (where $a$ is the orbital semi-major axis). Recently, Faigler et al. (2012) characterized seven non-eclipsing binaries thanks to these effects, but none of them present an orbital period longer than ten days. Assuming the same systems but with a orbital semi-major axis ten times larger, the beaming, reflexion, and ellipsoidal effects would present an amplitude at the level of the $100 \mathrm{ppm}, 2 \mathrm{ppm}, 1 \mathrm{ppm}$ (respectively), or below. We therefore consider that these effects can only marginally be used to distinguish secondary-only eclipsing binaries from out-of-transit variations, especially if the primary star is active.

\section{Estimation of the occurrence rate of secondary-only eclipsing binaries}

\subsection{Modeling the population of multiple stellar systems}

To evaluate the occurrence of this EB configuration, we performed a Monte Carlo simulation by considering $10^{6}$ binaries, 
A. Santerne et al.: The contribution of secondary eclipses as astrophysical false positives to exoplanet transit surveys

Table 1. 1: Fraction of eclipsing binaries in double and triple systems and transiting giant planets, among $10^{6}$ simulated systems, which present either the primary or secondary or both primary and secondary eclipse(s), as seen from the Earth. 2: Global occurrence rate for these systems, as reported in the literature. 3: Occurrence rate (fraction $\times$ overall likelihood $\times$ spectral type correction) of secondary-only, primary-only, and both primary and secondary eclipse(s) for the different configurations of system. 4: Number of KOIs that might be mimicked by a secondary-only system according to the Kepler-capability detection model of Fressin et al. (2013).

\begin{tabular}{|c|c|c|c|c|}
\hline 1. & Binary system & Hierarchical triple & Nonhierarchical triple & Giant planet \\
\hline Fraction of secondary-only & $0.126 \% \pm 0.004 \%$ & $0.227 \% \pm 0.005 \%$ & $0.391 \% \pm 0.006 \%$ & $0.091 \% \pm 0.003 \%$ \\
\hline Fraction of primary-only & $0.129 \% \pm 0.004 \%$ & $0.228 \% \pm 0.005 \%$ & $0.394 \% \pm 0.006 \%$ & $0.482 \% \pm 0.007 \%$ \\
\hline Fraction of primary and secondary & $0.633 \% \pm 0.008 \%$ & $2.153 \% \pm 0.015 \%$ & $3.632 \% \pm 0.019 \%$ & $1.703 \% \pm 0.013 \%$ \\
\hline Total fraction of primary and/or secondary & $0.888 \% \pm 0.016 \%$ & $2.608 \% \pm 0.025 \%$ & $4.417 \% \pm 0.031 \%$ & $2.276 \% \pm 0.023 \%$ \\
\hline 2. & Binary system & Hierarchical triple & Nonhierarchical triple & Giant planet \\
\hline Overall likelihood & $33 \% \pm 2 \%$ & $11 \% \pm 2 \% \times 76 \% \%^{\dagger}$ & $11 \% \pm 2 \% \times 24 \% \dagger$ & $9.7 \% \pm 1.3 \%$ \\
\hline 3. & Binary system & Hierarchical triple & Nonhierarchical triple & Giant planet \\
\hline Occurrence of secondary-only & $0.043 \% \pm 0.004 \%$ & $0.019 \% \pm 0.007 \%$ & $0.011 \% \pm 0.003 \%$ & $0.009 \% \pm 0.001 \%$ \\
\hline Occurrence of primary-only & $0.044 \% \pm 0.004 \%$ & $0.020 \% \pm 0.007 \%$ & $0.011 \% \pm 0.003 \%$ & $0.047 \% \pm 0.007 \%$ \\
\hline Occurrence of primary and secondary & $0.215 \% \pm 0.016 \%$ & $0.184 \% \pm 0.062 \%$ & $0.099 \% \pm 0.025 \%$ & $0.165 \% \pm 0.023 \%$ \\
\hline Total occurrence of primary and/or secondary & $0.302 \% \pm 0.022 \%$ & $0.223 \% \pm 0.075 \%$ & $0.120 \% \pm 0.031 \%$ & $0.221 \% \pm 0.031 \%$ \\
\hline 4. & Binary system & Hierarchical triple & Nonhierarchical triple & Giant planet \\
\hline Number of mimicked KOIs & $22.6 \pm 4.4$ & $10.0 \pm 2.7$ & $10.1 \pm 2.7$ & $0.4 \pm 0.4$ \\
\hline
\end{tabular}

Notes. ${ }^{(\dagger)}$ As reported by Raghavan et al. (2010); ${ }^{(\ddagger)}$ as reported by Mayor et al. (2011) for giant planets at any orbital period $\left(m_{\mathrm{p}} \sin i>100 M_{\oplus}\right)$.

hierarchical triple systems (where the inner-binary is composed of the two lowest mass star), and nonhierarchical triple systems (where the brightest star is member of the inner-binary) based on the statistics reported in Raghavan et al. (2010). We thus assumed the following distributions:

- log-normal distribution of orbital period $(P)$ either for the binary, inner-, and outer-binary of a triple system, centered at $\log _{10} P=5.03$ with a width of $\sigma_{\log _{10}^{P}}=2.28$ (Raghavan et al. 2010) ;

- distribution of primary mass of the Kepler targets, according to the Kepler Input Catalog (Brown et al. 2011), which is representative of a population of solar-type main-sequence stars outside of the galactic plane. After rejecting targets with $\log g<3.6$, these stars range in mass from $\sim 0.3 M_{\odot}$ to $\sim 2.1 M_{\odot}$, with a sharp maximum close to $1 M_{\odot}$;

- mass-ratio distributions following Fig. 16 of Raghavan et al. (2010). We do not consider brown dwarfs owing to the limitation of our isochrones and their relatively rare occurrence;

- sine distribution of orbital inclination (Figueira et al. 2012);

- uniform distribution of $\omega$ between $0^{\circ}$ and $360^{\circ}$;

- a circular orbit for systems with orbital periods less than ten days; a uniform distribution between a zero eccentricity and a linear upper-envelope ranging in eccentricity in [0.5; 0.95] and in period in $[10 ; 100]$ days (see Fig. 2). For periods longer than 100 days, we assumed a uniform distribution in the range [0;0.95] (see Fig. 2).

We limit the eccentricities to a reasonable maximum value $(e<$ $0.95)$ to avoid overestimating the fraction of secondary-only EB. The stability of triple systems was tested using Eq. (16) of Rappaport et al. (2013) and unstable systems were not allowed. Stellar radius were estimated using an isochrone of $1 \mathrm{Gyr}$ from the Dartmouth Stellar Evolution Database (Dotter et al. 2008). For each binary that satisfies Eqs. (1) and (2), we computed the secondary-eclipse depth with the PASTIS code (Díaz et al. 2013) for the Kepler bandpass as described in the previous section.

\subsection{Occurrence and comparison with other result}

The fractions of EBs found are reported in Table 1 for each type (secondary-only, primary-only, and both primary and secondary), and their distributions are displayed in Figs. 4 and discussed in Sect. 3.3. To compute the occurrence of each scenario, we multiplied the fraction (among our $10^{6}$ simulated cases) of such a scenario with the overall likelihood that a given star follow this scenario (Raghavan et al. 2010; Duchêne \& Kraus 2013). Following Fressin et al. (2013), we corrected the overall likelihood by the mass of the primary to reflect that massive stars are more common in multiple system than low-mass stars (see Fig. 12 in Raghavan et al. 2010). We find that $0.043 \% \pm 0.004 \%$ of stars are secondary-only EBs and $0.030 \% \pm 0.010 \%$ of stars are secondary-only EBs in triple systems. Considering only those that present a depth shallower than $3 \%$, we find an occurrence rate of secondary-only EB of $0.061 \% \pm 0.017 \%$ for primary of spectral type FGK IV-V. Uncertainties were estimated by considering the uncertainty of our simulation (assuming a Poisson noise) and the uncertainty on the occurrence of binaries from Raghavan et al. (2010). We also accounted for the uncertainty on the occurrence of the different hierarchies in triple systems (Raghavan et al. 2010). Allowing short-period EB (less than ten days) to be eccentric in our simulation, using the same prior distribution as for binaries with period longer than ten days, we found an upper occurrence of secondary-only EB of $0.082 \pm 0.025 \%$. Thus, considering the eccentricity of short-period binaries or not does not change the occurrence rate within $1-\sigma$.

Santerne et al. (2012) characterized two secondary-only eclipsing binaries using radial velocity observations with the SOPHIE spectrograph (Bouchy et al. 2009). These two false 
positives were observed in a selection of 46 close-in giant candidates with orbital periods of less than 25 days, transit depths deeper than $0.4 \%$, and a host star brighter than $K_{\mathrm{p}}=14.7$. From the MAST archive, we found 63542 dwarfs (with $\log g>4.0$ ) brighter than $K_{\mathrm{p}}=14.7$ observed by Kepler since 2009 . From our simulation, selecting only the secondary-only EB with orbital periods of less than 25 days and with eclipse depths between $0.4 \%$ and $3 \%$, we expect $4.3 \pm 0.5$ false positives in the Santerne et al. (2012) sample. This discrepancy might be explained either by some secondary-only EB in the eight candidates flagged with a vetting of four by Borucki et al. (2012) that were not observed with SOPHIE. This discrepancy might also be explained by some secondary-only EB that would have been identified as false positive prior to ground-based observations in the vetting process performed by the Kepler team. This discrepancy, based on small number statistics, might also reveal the overestimation of this false-positive scenario in our simulation.

Slawson et al. (2011) report an occurrence rate of detachedEB of $0.79 \%$ in the Kepler EB catalog which is significantly higher than our estimation listed in Table 1. First of all, we believe that the occurrence of EB in the Kepler catalog is slightly over estimated since it is composed of several confirmed planets, such as KIC9818381 also known as KOI-135 b/Kepler-43 b (Bonomo et al. 2012), KIC5728139 - KOI-206 b (Almenara et al. in prep.; Santerne et al. 2012), or Kepler-76 b (Faigler et al. 2013). Then, the Kepler EB catalog is composed of EB that do not involve the target star. Accounting for all EB from our simulation that present at least a primary or secondary eclipse in our model with a depth greater than $3 \%$, we found an occurrence of EB of $0.53 \% \pm 0.14 \%$ that is compatible at $1.8-\sigma$ with the occurrence of EB found in the Kepler field.

We tested the dependence of our results on the assumed prior distributions. We expect the period distribution to have a significant impact on the resulting occurrence, especially for the inner binary of the triple system. All inner binary of triple systems reported by Raghavan et al. (2010) present an orbital period of less than 100 days. Limiting the periods of such binaries to 100 days increases the fractions and occurrences of the hierarchical and nonhierarchical triple systems reported in Table 1 by factors of 1.43 and 1.46 (respectively). In that case, our value of the occurrence of EB would be in better agreement with the value from Slawson et al. (2011). The primarymass distribution is expected to significantly affect the results. Assuming the population of F-K dwarfs with $11<m_{R}<16$ located in each of the CoRoT eyes (Boisnard \& Auvergne 2006), as simulated with the Besançon galactic model (Robin et al. 2003), we found a lower occurrence of $20 \pm 9 \%$ and $16 \pm 6 \%$ for the center and anticenter fields, respectively. This might be explained by the fact that dwarfs in the CoRoT eyes are on average smaller than the selected Kepler targets, according to the Besançon galactic model. Thus, both their eclipse probability and binary occurrence rates are lower. Finally, assuming the eccentricity distribution reported by Raghavan et al. (2010) for binaries with periods below 1000 days, within our envelope displayed in Fig. 2, we find a lower occurrence of secondary-only EB of $0.039 \pm 0.012 \%$, at $1-\sigma$ from the occurrence rate found with a uniform distribution of eccentricity.

\subsection{Distributions of secondary-only eclipsing binaries}

Figure 4 displays the stacked distribution of depth and transit duration of the simulated binaries that present a secondary-only eclipse. The eclipse depth were estimated as the minimum point of the synthetic light curve. This was only possible due to the good sampling of the synthetic light curve (samping of $10^{-4}$ in phase). Eclipse durations were estimated using the following equation, from Winn (2010):

$T_{14}=\frac{P}{\pi} \sin ^{-1}\left[\frac{R_{1}}{a} \frac{\sqrt{\left(1+\frac{R_{2}}{R_{1}}\right)^{2}-b_{\mathrm{sec}}^{2}}}{\sin i}\right] \frac{\sqrt{1-e^{2}}}{1-e \sin \omega}$.

The orbital period, inclination, eccentricity, and argument of periastron distribution of secondary-only eclipsing binaries are displayed in Fig. 4. We note that secondary-only EB can mimic the transit depth of planet-candidate for the whole range of radius detectable by Kepler. However, secondary-only EB in binary system or nonhierarchical systems more likely mimic giant planets, while secondary-only EBs in hierarchical triple mimic planet candidates with the size of Neptune.

Secondary-only EB present eclipse duration with a median of $3.95 \mathrm{~h}$, while the median value for all the KOIs is $3.41 \mathrm{~h}$. Nevertheless, as shown in Fig. 4 and discussed in Sect. 2.2, secondary-only EB have an orbital period greater than about ten days for the vast majority of cases. The median value of transit duration among the KOIs with period of more than ten days is $4.65 \mathrm{~h}$, which is slightly longer than the one estimated for the secondary-only EB. As discussed in Sect. 2.3, the shorter duration of secondary-only eclipse might be interpreted as an eccentric transiting planet.

Due to the effect of tidal circularization for binaries with orbital period of fewer than ten days, we did not include eccentric binaries with such short orbital periods in our simulation. Secondary-only EB thus present a orbital period longer than ten days, with a median of $\sim 134$ days for a binary system, and $\sim 63$ days and 61 days for hierarchical and nonhierarchical triples, respectively. Accounting for the respective occurrence of the different configuration of multiple system, secondary-only EB have an orbital period with a median of 116 days, while the median period of the KOIs is 11 days. This new configuration of false positive is thus expected to be more frequent for longperiod candidates. The distribution of orbital inclination deduced from our simulation is not obviously different from the one observed in the KOIs ones, which are dominated by the geometrical transit/eclipse probability.

The eccentricity of secondary-only EB is the most important orbital parameter for the configuration of false positives presented in this paper. The eccentricity of secondary-only EB have relative high-eccentricity, with a median of $\sim 0.7$ ( 0.73 for those in binary system and 0.61 for those in triple system). As expected by Eqs. (1) and (2), we find a posterior distribution of argument of periastron in the range $\left(180^{\circ} ; 360^{\circ}\right)$, centered on $270^{\circ}$. These values of $\omega$ are the only ones that allow only the secondary eclipse to be seen.

\section{Occulting-only giant planets as false positive scenario}

\subsection{Modeling the population of giant planets}

We now consider the occultation of a giant planet instead of the secondary eclipse of a binary. We reproduce the previous simulation using the same distribution for $\omega, i$, and $R_{\star}$ as for binaries. We used the period distribution and eccentricity (within the envelope displayed in Fig. 2) distribution of giant planets discovered to date by radial velocity (for $m_{\mathrm{p}} \sin i>0.3 M_{\text {Jup }}$ ) as provided by the Exoplanet Data Explorer (Wright et al. 2011) 
A. Santerne et al.: The contribution of secondary eclipses as astrophysical false positives to exoplanet transit surveys

and the radius distribution of Kepler giant transiting candidates ${ }^{1}$ (for $6 R_{\oplus}<r_{\mathrm{p}}<22 R_{\oplus}$, Batalha et al. 2012). For each simulated giant planet, we computed the impact parameters of both the transit $\left(b_{\text {tr }}\right)$ and occultation $\left(b_{\text {occ }}\right)$. Then, we considered an occulting-only planet if (Winn 2010):

$$
\begin{gathered}
b_{\mathrm{tr}}=\frac{a}{R_{\star}} \cos (i)\left(\frac{1-e^{2}}{1+e \sin \omega}\right)>1+\frac{r_{\mathrm{p}}}{R_{\star}}, \\
b_{\mathrm{occ}}=\frac{a}{R_{\star}} \cos (i)\left(\frac{1-e^{2}}{1-e \sin \omega}\right)<1-\frac{r_{\mathrm{p}}}{R_{\star}},
\end{gathered}
$$

where $r_{\mathrm{p}}$ is the planetary radius. In the present case, we rejected grazing occultations that are too shallow to reproduce even an Earth-size transit, compared with the binary simulation for which we kept all the grazing eclipse. For each occultingonly giant planet, we computed the occultation depth $\left(\delta_{\text {occ }}\right)$, assuming no thermal emission from the planet (Rowe et al. 2006):

$\delta_{\text {occ }}=A_{\mathrm{g}}\left(\frac{r_{\mathrm{p}}}{a_{\mathrm{occ}}}\right)^{2}$,

where $A_{\mathrm{g}}$ is the geometric albedo, supposed to be 0.1 for the majority of close-in giant planets (Cowan \& Agol 2011), and

$a_{\mathrm{occ}}=a\left(\frac{1-e^{2}}{1-e \sin \omega}\right)$

is the separation between the star and the planet during the occultation.

\subsection{Results and comparison with other occurrences}

Assuming the occurrence rate of giant planets reported by Mayor et al. (2011), we found that $0.009 \% \pm 0.002 \%$ of solar-type stars should harbor a giant planet that only presents the occultation, as seen from the Earth. When accounting only for those who present an occultation deeper than $1 \mathrm{ppm}$, the occurrence decrease to $0.005 \pm 0.001 \%$. All the fractions and occurrences of transiting and/or occulting giant planets are listed in Table 1. As for the binary and triple, uncertainties were estimated by considering the uncertainty of our simulation assuming a Poisson noise and the uncertainty on the occurrence of giant planets from Mayor et al. (2011). These results can be compared with the expected $235 \times(1-19 \%) \sim 190$ giant transiting planets in the Kepler field (Batalha et al. 2012), after accounting for $19 \%$ of false positive (Fressin et al. 2013). Assuming 156000 stars observed by Kepler, we can expect from our result $331 \pm 44$ giant planets. This discrepancy (at 3.2- $\sigma$ ) might be explained by the difference in the occurrence rate of planets between the Kepler survey (Howard et al. 2012; Santerne et al. 2012; Fressin et al. 2013) and the radial velocity surveys (e.g. Mayor et al. 2011; Wright et al. 2012). Assuming the occurrence of giant planets from Fressin et al. (2013), i.e. $5.12 \% \pm 0.55 \%$, we expected Kepler to have found $175 \pm 19$ giants planets (including $~ 35$ grazing giant planets), in better agreement with the observed number of candidates.

\subsection{Distributions of occulting-only giant planet}

The distribution of occulting-only giant planets in occultation depth, duration, orbital period, inclination, eccentricity, and argument of periastron are displayed in Fig. 4. They first reveal

\footnotetext{
We assumed here that the $\sim 19 \%$ of false-positives (Fressin et al. 2013) do not significantly bias the radius distribution within the considered range of radii.
}

Table 2. Expected radius of KOIs mimicked by secondary-only eclipsing binary or occulting-only giant planets.

\begin{tabular}{lc}
\hline \hline Expected size of KOI & Number of mimicked KOIs \\
\hline Earth-size $\left(0.8-1.25 R_{\oplus}\right)$ & $0.2 \pm 0.2$ \\
Super-earth $\left(1.25-2 R_{\oplus}\right)$ & $1.2 \pm 1.0$ \\
Small neptunes $\left(2-4 R_{\oplus}\right)$ & $5.1 \pm 1.9$ \\
Large neptunes $\left(4-6 R_{\oplus}\right)$ & $10.5 \pm 2.8$ \\
Giant planets $\left(6-22 R_{\oplus}\right)$ & $26.0 \pm 4.5$ \\
\hline
\end{tabular}

that occulting-only giant planets are mimicking sub-Earth objects (like Kepler-37 b, Barclay et al. 2013), with depths up to a few tens of ppm, with the exception being for a much higher geometric albedo than considered here (e.g. Santerne et al. 2011). Then, this type of false positive (even if there are undiluted planets, their characteristics might be misinterpreted) would present a short transit with a median of about $1.7 \mathrm{~h}$ with a median orbital period of about ten days. They should present a moderate eccentricity with a median value of $\sim 0.3$.

\section{Discussion and conclusion}

We report in this paper a new configuration of false positives involving eclipsing binaries for which only the secondary eclipse occurs. By simulating three secondary-only eclipsing binaries presenting different apparent transit depths, we showed that this false positive can mimic a grazing planetary transit in an eccentric or circular orbit and thus pass unnoticed through a lightcurve inspection. We then simulated a population of binary and giant planets and found that $0.061 \% \pm 0.017 \%$ and $0.009 \% \pm$ $0.002 \%$ of solar-type stars harbor a secondary-only eclipsing binary or transiting giant planet, respectively. To evaluate the impact of this configuration of false positives in the context of the Kepler mission, we simulated secondary-only eclipsing binaries and occulting-only giant planets using the Kepler-capability detection model of Fressin et al. (2013). We find that up to $43.1 \pm 5.6 \mathrm{KOIs}$ can be mimicked by this configuration of false positives. This corresponds to $1.9 \pm 0.2 \%$ of the total KOIs identified by Batalha et al. (2012), thereby re-evaluating the global FPP of the Kepler mission from $9.4 \pm 0.9 \%$ (Fressin et al. 2013) to $11.3 \pm 1.1 \%$. These scenarios of false positives do not change the global FPP reported by Fressin et al. (2013) significantly but should be taken into account when validating planet candidates (as in Morton 2012). The detailed numbers of KOIs mimicked by each scenario considered in this paper are listed in Table 1, and the different apparent sizes of the mimicked KOIs are listed in Table 2. These results show that this configuration of false positive scenario tends to mimic giant planets. These false positives scenarios are more likely to occur for the long-period KOIs, as discussed in Sect. 3.3.

As displayed in Fig. 4 the expected depths of an occultingonly giant planet is less than a few tens of ppm, which only concerns the shallowest part of the Kepler candidates. Only $0.4 \pm 0.4 \mathrm{KOI}$ is expected to be mimicked by this scenario. This type of false positive is expected to be more significant in the next-generation space-based transit surveys, like PLATO (Rauer \& Catala 2012), whose objective is to reach the ppm-level accuracy for most of the targets, observing much brighter stars than CoRoT and Kepler.

We stress that our simulations strongly depend on our current knowledge of the binary population, which is mostly based on the results from Raghavan et al. (2010). Even if the authors 
performed a rigorous characterization of multiplicity of solartype stars within $25 \mathrm{pc}$, their results are based on a relatively small statistics involving about 200 binaries and 33 triple systems. Moreover, the stellar multiplicity in transit-survey fields might be different than for the very local neighborhood. A careful statistical analysis of the thousands of binaries (eclipsing or presenting some beaming, ellipsoidal, or reflexion effects; Faigler et al. 2012) observed in the Kepler and CoRoT fields will permit an even better understanding of the stellar multiplicity in the galaxy. The ESA Gaia mission or LSST survey will also be able to provide a large number of binaries in different regions of the MilkyWay which will greatly strengthen the statistics on binary populations (Eyer et al. 2012). This study of stellar multiplicity is also important for improving the priors used for false positives in the planet-validation process.

For observed secondary-only eclipsing binaries, the reference epoch of eclipse matches the secondary eclipse. It is thus expected that any radial velocity follow-up will find a significant variation in antiphase with the ephemeris (see discussion about KOI-419 and KOI-698 in Santerne et al. 2012). Therefore, this might explain the nine cases of high-amplitude, radial-velocity variation in antiphase that were found in the first fields of CoRoT as reported by Cabrera et al. (2009), Erikson et al. (2012), Carone et al. (2012), and Cavarroc et al. (2012). Interestingly, four of these candidates present transit-like events with periods longer than or about ten days, as expected, but three present a period of about six days and two others have a period of about two days. If these short-period antiphase candidates are actually secondary-only EB, this would imply that we have underestimated the occurrence of this false-positive scenario in the short-period range. If they are finally more common than we assumed here, these short-period eccentric EB for which only the secondary eclipse is seen should also be present in the groundbased transit surveys, such as Super-WASP (Cameron et al. 2007; Triaud 2011), HATNet (Bakos et al. 2007), and NGTS (Chazelas et al. 2012).

Acknowledgements. We thank the anonymous referee for fruitful comments that helped us to substantially improve the quality of this paper. We also thank J. Johnson and T. Morton for their constructive comments about this paper. A.S., P.F., and N.C.S. acknowledge the support by the European Research Council/European Community under the FP7 through Starting Grant agreement number 239953. NCS also acknowledges the support from Fundação para a Ciência e a Tecnologia (FCT) in the form of grant reference PTDC/CTEAST/098528/2008. RFD is supported by the CNES.

\section{References}

Abt, H. A. 2005, ApJ, 629, 507

Allard, F., Homeier, D., \& Freytag, B. 2012, IAU Symp., 282, 235 Almenara, J. M., Deeg, H. J., Aigrain, S., et al. 2009, A\&A, 506, 337 Bakos, G. Á., Noyes, R. W., Kovács, G., et al. 2007, ApJ, 656, 552 Barclay, T., Rowe, J. F., Lissauer, J. J., et al. 2013, Nature, 494, 452 Batalha, N. M., Rowe, J. F., Bryson, S. T., et al. 2013, ApJS, 204, 24 Boisnard, L., \& Auvergne, M. 2006, ESA SP, 1306, 19
Bonomo, A. S., Santerne, A., Alonso, R., et al. 2010, A\&A, 520, A65 Bonomo, A. S., Hébrard, G., Santerne, A., et al. 2012, A\&A, 538, A96 Borucki, W. J., Koch, D. G., Batalha, N., et al. 2012, ApJ, 745, 120

Bouchy, F., Hébrard, G., Udry, S., et al. 2009, A\&A, 505, 853

Brown, T. M., Latham, D. W., Everett, M. E., \& Esquerdo, G. A. 2011, AJ, 142, 112

Bulut, I., \& Demircan, O. 2007, MNRAS, 378, 179

Cabrera, J., Fridlund, M., Ollivier, M., et al. 2009, A\&A, 506, 501

Cameron, A. C. 2012, Nature, 492, 48

Cameron, A. C., Bouchy, F., Hébrard, G., et al. 2007, MNRAS, 375, 951

Carone, L., Gandolfi, D., Cabrera, J., et al. 2012, A\&A, 538, A112

Cavarroc, C., Moutou, C., Gandolfi, D., et al. 2012, Ap\&SS, 337, 511

Chazelas, B., Pollacco, D., Queloz, D., et al. 2012, Proc. SPIE, 8444, 10

Claret, A., Hauschildt, P. H., \& Witte, S. 2012, A\&A, 546, A14

Cowan, N. B., \& Agol, E. 2011, ApJ, 729, 54

Dawson, R. I., \& Johnson, J. A. 2012, ApJ, 756, 122

Dawson, R. I., Johnson, J. A., Morton, T. D., et al. 2012, ApJ, 761, 163

Devor, J., Charbonneau, D., O’Donovan, F. T., Mandushev, G., \& Torres, G. 2008, AJ, 135, 850

Díaz, R. F., Almenara, J.-M., Santerne, A. et al. 2013, MNRAS, submitted

Dotter, A., Chaboyer, B., Jevremović, D., et al. 2008, ApJS, 178, 89

Duchêne, G., \& Kraus, A. 2013, ARA\&A, in press [arXiv: 1303.3028]

Duquennoy, A., \& Mayor, M. 1991, A\&A, 248, 485

Erikson, A., Santerne, A., Renner, S., et al. 2012, A\&A, 539, A14

Eyer, L., Dubath, P., Mowlavi, N., et al. 2012, IAU Symp., 282, 33

Faigler, S., Mazeh, T., Quinn, S. N., Latham, D. W., \& Tal-Or, L. 2012, ApJ 746,185

Faigler, S., Tal-Or, L., Mazeh, T., Latham, D. W., \& Buchhave, L. A. 2013, ApJ, 771,26

Figueira, P., Marmier, M., Boué, G., et al. 2012, A\&A, 541, A139

Fressin, F., Torres, G., Pont, F., et al. 2012a, ApJ, 745, 81

Fressin, F., Torres, G., Rowe, J. F., et al. 2012b, Nature, 482, 195

Fressin, F., Torres, G., Charbonneau, D., et al. 2013, ApJ, 766, 81

Halbwachs, J. L., Mayor, M., Udry, S., \& Arenou, F. 2003, A\&A, 397, 159

Howard, A. W., Marcy, G. W., Bryson, S. T., et al. 2012, ApJS, 201, 15

Kozai, Y. 1962, AJ, 67, 591

Kreiner, J. M., Kim, C.-H., \& Nha, I.-S. 2001, An Atlas of O-C Diagrams of Eclipsing Binary Stars (Cracow, Poland: Wydawnictwo Naukowe Akademii Pedagogicznej)

Mayor, M., Marmier, M., Lovis, C., et al. 2011, A\&A, submitted [arXiv: 1109.2497]

Mazeh, T., \& Faigler, S. 2010, A\&A, 521, L59

Morton, T. D. 2012, ApJ, 761, 6

Morton, T. D., \& Johnson, J. A. 2011, ApJ, 738, 170

Pourbaix, D., Tokovinin, A. A., Batten, A. H., et al. 2004, A\&A, 424, 727

Perryman, M. A. C., Lindegren, L., Kovalevsky, J., et al. 1997, A\&A, 323, L49

Rappaport, S., Deck, K., Levine, A., et al. 2013 [arXiv: 1302.0563]

Raghavan, D., McAlister, H. A., Henry, T. J., et al. 2010, ApJS, 190, 1

Rauer, H., \& Catala, C. 2012, EGU General Assembly Conf. Abstr., 14, 7033

Robin, A. C., Reylé, C., Derrière, S., \& Picaud, S. 2003, A\&A, 409, 523

Rowe, J. F., Matthews, J. M., Seager, S., et al. 2006, ApJ, 646, 1241

Santerne, A., Bonomo, A. S., Hébrard, G., et al. 2011, A\&A, 536, A70

Santerne, A., Díaz, R. F., Moutou, C., et al. 2012, A\&A, 545, A76

Shporer, A., Jenkins, J. M., Rowe, J. F., et al. 2011, AJ, 142, 195

Slawson, R. W., Prša, A., Welsh, W. F., et al. 2011, AJ, 142, 160

Southworth, J. 2008, MNRAS, 386, 1644

Tingley, B., Bonomo, A. S., \& Deeg, H. J. 2011, ApJ, 726, 112

Torres, G., Fressin, F., Batalha, N. M., et al. 2011, ApJ, 727, 24

Triaud, A. H. M. J. 2011, Ph.D. Thesis

Winn, J. N. 2010 [arXiv: 1001.2010 ]

Wright, J. T., Fakhouri, O., Marcy, G. W., et al. 2011, PASP, 123, 412

Wright, J. T., Marcy, G. W., Howard, A. W., et al. 2012, ApJ, 753, 160

Zahn, J.-P. 1977, A\&A, 57, 383

Zahn, J.-P. 1989, A\&A, 220, 112 